\title{
EXPERIMENTOS DIDÁTICOS EM QUÍMICA ANALÍTICA ENVOLVENDO SEPARAÇÃO DE FASES E PRÉ- CONCENTRAÇÃOO
}

Clésia C. Nascentes e Marco A. Z. Arruda*

Departamento de Química Analítica, Instituto de Química, Universidade Estadual de Campinas, CP 6154, 13083-970 Campinas -SP Nelson Maniasso"

Centro de Energia Nuclear na Agricultura, Universidade de São Paulo, CP 96, 13400-970 Piracicaba - SP

Recebido em 15/1/01; aceito em 5/12/01

\begin{abstract}
DIDACTIC EXPERIMENTS IN ANALYTICAL CHEMISTRY INVOLVING PHASE SEPARATIONS AND PRECONCENTRATION. The aim of this work was to propose two different didactic experiments, which can be used in practical classes of analytical chemistry courses. More flexible experiments related to the theme, giving some options to the instructor are proposed. In this way, the Experiment 1 was divided in two parts. In the first one, the visualization of two distinct phases separation is emphasized: the rich and the poor phases in surfactant. In the second part, the metal pre-concentration (Co as example) is emphasized. The Experiment 2 has three different parts. In the first one, the complex formation is pointed out, in the second one, the $\mathrm{pH}$ influence is shown and in the last one, the influence of the complexation time is demonstrated.
\end{abstract}

Keywords: separations; preconcentration; cloud point; atomic spectrometry.

\section{INTRODUÇÃO}

Nos métodos analíticos, as etapas que envolvem separação de fases são empregadas, geralmente, visando a melhora em sensibilidade e/ou em seletividade ${ }^{1}$. Estas etapas envolvem, às vezes, o emprego de solventes orgânicos, sendo que o uso destes pode acarretar certos problemas ambientais, decorrentes da alta toxicidade dos mesmos. Devido a isto, existe a necessidade de desenvolver novas metodologias, as quais visem a substituição destes solventes por compostos biodegradáveis ${ }^{2}$, principalmente quando se considera a condução de uma aula prática.

Neste sentido, este trabalho aborda experimentos que permitem visualizar didaticamente o fenômeno da separação de fases em uma solução homogênea. Isso ocorre com o emprego de tensoativos na forma de ambientes micelares, utilizando a extração com separação de fases denominada Ponto Nuvem ${ }^{3-5}$ (do inglês, "Cloud Point"). Assim, supre-se, de forma segura, os problemas já mencionados quanto ao uso dos solventes orgânicos.

Os tensoativos foram escolhidos por serem biodegradáveis e também pela sua ampla utilização na prospecção de petróleo, como componente de óleos automotivos, em fármacos, em produtos de higiene pessoal como xampus, em cosméticos, e em produtos domésticos como amaciantes, detergentes, entre outros ${ }^{6,7}$.

O objetivo deste trabalho foi propor métodos alternativos, envolvendo a extração no Ponto Nuvem de analitos na forma de complexos, para serem aplicados em aulas práticas de química analítica, enfatizando alguns conceitos tais como complexação, pré-concentração, extração e separação de fases.

Desta forma, este trabalho é dividido em 2 partes principais, apresentando-se sugestões de aulas práticas, envolvendo os temas descritos acima. A forma de apresentação das práticas possibilita ampla discussão em sala de aula, já que cada experimento pode ser direcionado de acordo com a proposta do professor, com o tema curricular a ser abordado, e com o tempo disponível para sua realização. Assim sendo, o fator chave para o êxito dos experimentos propostos é, sem dúvida, a criatividade do docente, já que a aula prática pode ser moldada de acordo com as suas necessidades e/ou com as possibilidades da instituição de ensino.

\section{O que é Ponto Nuvem? Como ocorre a separação de fases?}

Os tensoativos (surfactantes ${ }^{\mathrm{a}}$ ) possuem a capacidade de modificar algumas propriedades reacionais visando melhora na sensibilidade e/ou seletividade do método analítico, sendo que as principais características do seu uso estão relacionadas à formação de ambientes organizados, também conhecidos como ambientes micelares ${ }^{6,8}$.

As micelas são definidas como agregados moleculares, possuidoras de regiões estruturais hidrofílica e hidrofóbica, que dinamica e espontaneamente se associam em meio aquoso, a partir de uma determinada concentração crítica (CMC - concentração micelar crítica), formando grandes agregados moleculares de dimensões coloidais. Abaixo da CMC, o tensoativo está predominantemente na forma monomérica ${ }^{9}$. As micelas são termodinamicamente estáveis, facilmente reprodutíveis e são destruídas pela diluição com água, quando a concentração do tensoativo ficar abaixo da $\mathrm{CMC}^{3}$.

Ambientes organizados na forma de micelas normal ou reversa, microemulsões ou vesículas ${ }^{3}$, são empregados em praticamente todos os campos da química analítica, visando a melhora das características analíticas dos métodos já existentes e/ou o desenvolvimento de novas metodologias.

A aplicação de tensoativos nos processos de separação em duas fases isotrópicas, fenômeno este denominado Ponto $\mathrm{Nuvem}^{3,4}$, tem

\footnotetext{
*e-mail: zezzi@iqm.unicamp.br

\# Endereço permanente: Departamento de Geoquímica, Universidade Federal Fluminense, Campus do Valonguinho, 24020-007 Niterói-RJ

a A definição da palavra surfactante é baseada na contração da expressão em inglês que descreve "surface-active agents". Estes proporcionam uma superfície ativa, devido à concentração de determinadas espécies em uma região de interface: ar-água, óleo-água ou sólido-líquido.
} 
sido reportada em substituição às metodologias tradicionais (resinas, extração líquido-líquido, etc.) em diversas áreas como: ambiental, biotecnológica e de saúde pública ${ }^{10-12}$.

O fenômeno Ponto Nuvem ${ }^{10-12}$ ocorre quando tensoativos (não iônicos ou anfóteros) em quantidades acima da CMC e aquecidos à uma determinada temperatura ${ }^{13,14}$, separam-se em duas fases isotrópicas (a solução começa subitamente a ficar turva, devido ao decréscimo de solubilidade do tensoativo em água). Esta separação de fases, parece estar associada à existência de micelas compostas por grandes agregados moleculares em solução. Uma outra possibilidade da ocorrência do fenômeno Ponto Nuvem se relaciona com o emprego de tensoativos aniônicos quando a concentração ácida do meio é elevada ${ }^{15}$.

Como ocorre a separação de fases no Ponto Nuvem, este pode ser empregado para a extração e pré-concentração de metais, já que duas fases são formadas neste processo: uma delas aquosa e contendo uma pequena quantidade de tensoativo (abaixo da CMC), denominada fase pobre e, a outra, extremamente concentrada em tensoativo e contendo os componentes extraídos da solução (denominada fase rica $^{5,16}$.

As principais características e/ou fatores relativos ao emprego de tensoativos na pré-concentração/separação, em comparação a outros métodos, foram amplamente discutidos na literatura ${ }^{16,17}$, e os seguintes aspectos devem ser considerados:

- O tensoativo deve possuir capacidade natural de extrair o analito

para a fase rica, sendo que o analito pode ser extraído em pequenos volumes desta fase;

- A separação das fases pode depender da hidrofobicidade do complexo formado com o analito, permitindo o uso de vários processos como, por exemplo, a extração líquido-líquido;

- O processo de separação das fases é reversível;

- Os tensoativos empregados não são tóxicos, voláteis ou inflamáveis; são de menor periculosidade quando comparados aos solventes orgânicos; requerem poucos miligramas e seu custo é geralmente baixo.

Desta forma, a separação de fases com o uso de tensoativos pode promover um elevado fator de pré-concentração, propiciando maior sensibilidade e possibilitando, muitas vezes, melhora em seletividade.

\section{PARTE EXPERIMENTAL}

\section{Equipamentos e acessórios}

- Espectrômetro de absorção atômica com chama AAnalyst 300 Perkin-Elmer (Norwalk, CT, EUA), equipado com lâmpada de deutério para a correção de fundo. Lâmpada de cátodo oco (Perkin-Elmer, Darmstadt, Alemanha) de Co foi empregada como fonte de radiação. As condições experimentais utilizadas na determinação de Co foram as recomendadas pelo fabricante.

- Centrífuga Nova Técnica modelo NT 811.

- Tubos de ensaio de $20 \mathrm{~mL}$.

\section{Reagentes e soluções}

Todas as soluções foram preparadas utilizando-se água deionizada e reagentes de grau analítico (Merck, Darmstadt, Alemanha).

Nos experimentos foram empregados os seguintes reagentes: 5Br-PADAP (2-(5-bromo-2-piridilazo)5-dietilaminofenol), PAN (1(2-piridilazo)2-naftol) e PAR (sal monossódico de 4-(2-piridilazo) resorsinol). Estes reagentes foram empregados como complexantes. Ainda, os tensoativos aniônicos, SDS - dodecilsulfato de sódio e SDSA - sal sódico do ácido dodecanosulfônico, foram empregados para a separação de fases. O tensoativo não-iônico Triton X-100, foi utilizado para a solubilização dos complexantes, atuando também na separação das fases, onde é formada uma micela mista.

As soluções de 5-Br-PADAP e PAR foram preparadas dissolvendo-se $25 \mathrm{mg}$ dos respectivos reagentes em $25 \mathrm{~mL}$ de Triton X-100 $4 \% \mathrm{v} / \mathrm{v}$ e completando-se o volume para $100 \mathrm{~mL}$ com água deionizada.

A solução de PAN foi preparada dissolvendo-se $25 \mathrm{mg}$ em 25 $\mathrm{mL}$ de etanol $+25 \mathrm{~mL}$ de Triton X-100 4\% v/v e completando-se o volume para $100 \mathrm{~mL}$ com água deionizada.

Os tensoativos SDS e SDSA foram empregados na forma sólida.

Soluções padrão de 10 e $100 \mathrm{mg} \mathrm{L}^{-1}$ Co foram preparadas a partir de uma solução estoque de $1000 \mathrm{mg} \mathrm{L}^{-1}$ Co [ampola Titrisol Merck] em $\mathrm{HNO}_{3} 1 \%$ v/v. A solução de $10 \mathrm{mg} \mathrm{L}^{-1} \mathrm{Co}$ foi utilizada como amostra para a pré-concentração e a de $100 \mathrm{mg} \mathrm{L}^{-1}$ Co para a curva de calibração.

Para a curva analítica de calibração $\left(0,5-3,0 \mathrm{mg} \mathrm{L}^{-1}\right)$, aos volumes adequados a partir da solução estoque de $100 \mathrm{mg} \mathrm{L}^{-1} \mathrm{Co}$, foram adicionados SDS (de 0,5 a 3,0 \% m/v, dependendo do experimento), $0,1 \%$ v/v de Triton X-100, etanol na mesma concentração encontrada nas micelas (50\% v/v) e o volume completado com $\mathrm{HCl} 1 \mathrm{~mol} \mathrm{~L}^{-1}$. Isto foi feito no intuito de evitar/minimizar efeitos de matriz indesejáveis, bem como problemas com a nebulização/ atomização. No entanto, devido à presença do etanol, a micela não era formada e a pré-concentração não era realizada para a obtenção da curva de calibração.

\section{Procedimento}

\section{Experimento 1}

\section{Primeira parte: Visualização da separação de fases}

Deve-se salientar que em todos os experimentos envolvidos neste trabalho, o volume final totaliza $20 \mathrm{~mL}$. Desta forma, as concentrações dos reagentes devem ser relacionadas a esse volume.

Será enfatizada nesta parte a visualização da separação das fases [uma rica, parte superior do tubo de ensaio, e a outra pobre em tensoativo, parte inferior do tubo de ensaio (vide Figura 1)]. Para este experimento serão usados 5 tubos de ensaio contendo somente água e SDSA $1 \%$ m/v. Para aumentar a hidrofobicidade do tensoativo na solução, serão adicionados diferentes volumes de ácido clorídrico $(1: 1 \mathrm{v} / \mathrm{v})$ para obter concentrações finais entre 1 e $5 \mathrm{~mol} \mathrm{~L}^{-1}$.

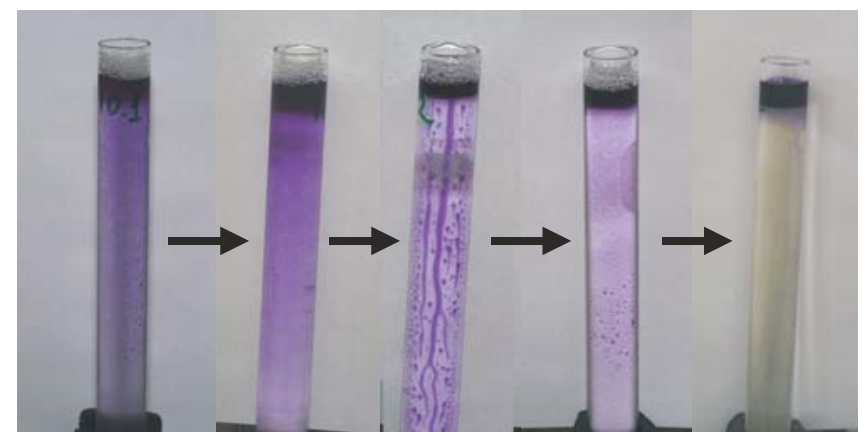

Figura 1. Seqüência de fotos da separação de fases obtidas para préconcentração de Co através do Ponto Nuvem. Fase colorida (superior) e transparente (inferior): denominadas de fase rica e fase pobre em tensoativo, respectivamente. As condições experimentais foram aquelas obtidas a partir do Experimento 1, segunda parte - pré-concentração. O tempo total envolvido, após a adição do $\mathrm{HCl}$, foi ca. de $3 \mathrm{~min}$ 
Este experimento serve para verificar a ocorrência, ou não, da separação de fases em um sistema homogêneo, devida a concentração do ácido.

A sequiência analítica para esta parte do experimento segue abaixo:

- $16,7-3,3 \mathrm{~mL}$ de $\mathrm{H}_{2} \mathrm{O}$

- $1 \% \mathrm{~m} / \mathrm{v}$ SDSA (agitar por $60 \mathrm{~s}$ )*

- 3,3 - 16,7 $\mathrm{mL}$ de $\mathrm{HCl}(1: 1 \mathrm{v} / \mathrm{v})^{*}$

- Homogeneizar a solução

- Centrifugar por 10 min a \pm 2500 rpm e observar a solução resultante.

* Antes de agitar recomenda-se vedar os tubos com parafilme. Esta observação é válida para todas as seqüências empregadas.

Todos os experimentos poderão ser feitos em réplicas, de acordo com a característica da aula e a disponibilidade de materiais e de tempo.

\section{Segunda parte: Pré-concentração}

A segunda parte deste experimento enfatiza a pré-concentração e a aplicação de alguns conceitos básicos, frequentemente empregados em química analítica, e relacionados com as características analíticas de um método. Serão testadas diferentes concentrações de SDS, de modo a se verificar a influência das mesmas na eficiência da préconcentração e no tamanho da fase rica em tensoativo.

A seqüência analítica para esta parte do experimento segue abaixo: - 4,7 $\mathrm{mL}$ de $\mathrm{H}_{2} \mathrm{O}$

- $6,25 \times 10^{-4} \% \mathrm{~m} / \mathrm{v}$ de 5-Br-PADAP $(0,5 \mathrm{~mL}$ da solução de 5 -BrPADAP $0,025 \% \mathrm{~m} / \mathrm{v}$ )

- $0,5 \mathrm{mg} \mathrm{L}^{-1}$ analito $\left(1 \mathrm{~mL}\right.$ a partir de uma solução padrão de $10 \mathrm{mg} \mathrm{L}^{-1}$ $\mathrm{Co})$

- ajustar pH a 9,0 (colocando 0,5 mL de $\mathrm{NaOH} \mathrm{0,1} \mathrm{mol} \mathrm{L}^{-1}$ )

- $0,5-3,0 \% \mathrm{~m} / \mathrm{v}$ SDS (agitar por $60 \mathrm{~s}$ )

- $13,3 \mathrm{~mL}$ de $\mathrm{HCl}(1: 1 \mathrm{v} / \mathrm{v})$

- Homogeneizar a solução.

- Centrifugar por 10 min a \pm 2500 rpm

- Determinar o tamanho das fases rica e pobre e separar a fase rica para separação do Co.

Após a separação, a fase rica em tensoativo é analisada por espectrometria de absorção atômica com chama (FAAS), sendo o cobalto determinado. Para isso, deve-se proceder à retirada da fase rica em tensoativo do tubo de ensaio. Isto deve ser feito de forma cuidadosa, evitando a retirada da fase pobre juntamente com a fase rica e a resolubilização das fases. A retirada da fase rica pode ser realizada com o uso de uma micro-pipeta ou, simplesmente, com uma pipeta de Pasteur de ponta bem fina. A fase rica é transferida para um outro recipiente, para permitir a introdução do capilar do nebulizador do instrumento de absorção atômica, uma vez que, introduzindo o capilar diretamente no tubo de ensaio, existe a possibilidade de introduzir, também, a fase pobre no espectrômetro.

Caso a fase rica esteja muito densa, a mesma pode ser diluída com etanol p.a. ou comercial ou, ainda, com HCldiluído (dependendo do objetivo da prática), sendo que o fator de diluição pode ficar a critério do docente (explorando características analíticas relacionadas aos limites de detecção e quantificação, bem como fundamentos sobre resultados analíticos, adequando o fator de diluição em relação à curva analítica de calibração).

\section{Experimento 2}

Primeira parte: Formação de complexos e equilíbrio químico

O segundo experimento se constitui numa sequiência lógica do primeiro, enfatizando temas como formação de complexo e equilí- brio químico na extração do analito pelo tensoativo. Neste sentido, serão estudados diferentes tipos de complexantes (PAR, PAN e 5Br-PADAP) na extração do analito, bem como será avaliada a ausência do complexante.

A seqüência analítica para esta parte do experimento segue abaixo:

- $4,7 \mathrm{~mL}$ de $\mathrm{H}_{2} \mathrm{O}$

- $6,25 \times 10^{-4} \% \mathrm{~m} / \mathrm{v}$ do complexante $(0,5 \mathrm{~mL}$ das soluções de $5-\mathrm{Br}$ PADAP ou PAR ou PAN) ou mais $0,5 \mathrm{~mL}$ de $\mathrm{H}_{2} \mathrm{O}$ (situação sem complexante)

- $0,5 \mathrm{mg} \mathrm{L}^{-1}$ analito ( $1 \mathrm{~mL}$ a partir de uma solução padrão de $10 \mathrm{mg} \mathrm{L}^{-1}$ Co)

- ajustar pH a 9,0 (colocando 0,5 mL de $\mathrm{NaOH} \mathrm{0,1} \mathrm{mol} \mathrm{L}^{-1}$ )

- $1,0 \% \mathrm{~m} / \mathrm{v}$ SDS (agitar por $60 \mathrm{~s}$ )

- $13,3 \mathrm{~mL}$ de $\mathrm{HCl}(1: 1 \mathrm{v} / \mathrm{v})$

- Homogeneizar a solução

- Centrifugar por $10 \mathrm{~min} \mathrm{a} \pm 2500 \mathrm{rpm}$

- Determinar o tamanho das fases rica e pobre e separar a fase rica para determinação de Co.

\section{Segunda parte: Influência do pH na formação do complexo}

Depois da definição do agente complexante e com o pH de complexação já estabelecido na literatura ${ }^{18}$, pode-se verificar a influência deste parâmetro na complexação, simplesmente não adicionando o $\mathrm{NaOH}$ para o ajuste do $\mathrm{pH}$. Deve-se destacar que o $\mathrm{pH}$ em que ocorre a complexação, não afeta a separação das fases isotrópicas. A alteração do $\mathrm{pH}$ que proporciona a separação de fases é obtida com a adição de $\mathrm{HCl}$ na última etapa do processo, ou seja, depois da interação complexo-surfactante. Desta forma, pretende-se explorar a influência do $\mathrm{pH}$ do meio reacional na formação de complexos. Isto pode ser comprovado medindo-se o $\mathrm{pH}$ em cada meio e estabelecendo os diferentes fatores de concentração para cada situação estudada.

A seguir, têm-se a sequiência analítica para esta parte do experimento:

- 4,7 mL de $\mathrm{H}_{2} \mathrm{O}$

- 6,25 x $10^{-4} \% \mathrm{~m} / \mathrm{v}$ de 5 -Br-PADAP $(0,5 \mathrm{~mL}$ da solução de $5-\mathrm{Br}$ PADAP $0,025 \% \mathrm{~m} / \mathrm{v}$ )

- $0,5 \mathrm{mg} \mathrm{L}^{-1}$ analito (1 $\mathrm{mL}$ a partir de uma solução padrão de $10 \mathrm{mg} \mathrm{L}^{-1}$ Co)

- sem ajuste de $\mathrm{pH}$ (ao redor 2,5) e com ajuste do $\mathrm{pH}$ para 9,0 (colocando $0,5 \mathrm{~mL}$ de $\mathrm{NaOH} 0,1 \mathrm{~mol} \mathrm{~L}^{-1}$ )

- $1,0 \% \mathrm{~m} / \mathrm{v}$ SDS (agitar por $60 \mathrm{~s}$ )

- 13,3 mL de $\mathrm{HCl}(1: 1 \mathrm{v} / \mathrm{v})$

- Homogeneizar a solução

- Centrifugar por $10 \mathrm{~min}$ a $\pm 2500 \mathrm{rpm}$

- Determinar o tamanho das fases rica e pobre e separar a fase rica para determinação de Co.

\section{Terceira parte: Influência do tempo de complexação}

A terceira etapa deste segundo experimento, consiste em estudar a influência do tempo de complexação. Estes experimentos foram realizados seguindo a mesma sequiência do anterior, porém, a adição do tensoativo foi realizada em diferentes tempos (0 - $60 \mathrm{~min})$.

Aqui, apresenta-se a seqüência analítica para esta parte do experimento:

- $4,7 \mathrm{~mL}$ de $\mathrm{H}_{2} \mathrm{O}$

- $6,25 \times 10^{-4} \% \mathrm{~m} / \mathrm{v}$ de 5 -Br-PADAP $(0,5 \mathrm{~mL}$ da solução de 5 -BrPADAP $0,025 \% \mathrm{~m} / \mathrm{v}$ )

- $0,5 \mathrm{mg} \mathrm{L}^{-1}$ analito (1 mL a partir de uma solução padrão de $10 \mathrm{mg} \mathrm{L}^{-1}$ Co) 
- ajustar pH a 9,0 (colocando 0,5 $\mathrm{mL}$ de $\mathrm{NaOH} 0,1 \mathrm{~mol} \mathrm{~L}^{-1}$ )

- intervalo de espera 0 - $60 \mathrm{~min}$

- $1,0 \% \mathrm{~m} / \mathrm{v}$ SDS (agitar $60 \mathrm{~s}$ )

- $13,3 \mathrm{~mL}$ de $\mathrm{HCl}(1: 1 \mathrm{v} / \mathrm{v})$

- Homogeneizar a solução

- Centrifugar por 10 minutos a $\pm 2500 \mathrm{rpm}$

- Determinar o tamanho das fases rica e pobre e separar a fase rica para determinação de Co.

\section{RESULTADOS E DISCUSSÃO}

\section{Experimento 1}

\section{Primeira parte: Visualização da separação de fases}

Na primeira parte do Experimento 1, objetivou-se a visualização da separação das fases isotrópicas (uma rica e a outra pobre em tensoativo). Para tal, foi empregado o tensoativo SDSA (tensoativo aniônico), o qual, após a adição do ácido na concentração adequada, permitiu a separação das fases quase que instantaneamente. Desta forma, os resultados com a adição de diferentes concentrações de ácido podem ser observados na Tabela 1, indicando claramente que há a necessidade de uma concentração ácida final entre 3 e $4 \mathrm{~mol} \mathrm{~L}^{-1}$, para que ocorra a separação das fases dentro de um tempo adequado para uma aula prática. Estes resultados corroboram com aqueles já descritos na literatura ${ }^{19,20}$. Estas fases são oriundas de uma solução homogênea, sendo que a sua separação pode ser explicada devido ao aumento da hidrofobicidade do tensoativo na solução. Esta condição pode ser melhor visualizada nas concentrações de $\mathrm{HCl}$ entre 3 $4 \mathrm{~mol} \mathrm{~L}^{-1}$. Apesar do efeito da temperatura ser importante na separação de fase ${ }^{13,14}$, influenciando esta separação, este efeito não foi estudado, uma vez que somente com o emprego do ácido era conseguida a separação das fases em poucos minutos. Deve-se comentar que com o aumento da concentração do ácido $\left(5 \mathrm{~mol} \mathrm{~L}^{-1}\right), \mathrm{e}$ até 4 h de observação, o que se notava era que a solução tornava-se opaca. Este fenômeno pode ser visualizado no trabalho de Casero et $a l .{ }^{15}$, onde é apresentado um diagrama de fases, no qual aumentando-se a concentração do ácido ocorre uma região que os autores chamam de região sólida (formação de precipitado). Apesar do mecanismo ainda não ter sido elucidado, supõe-se que ao elevar ainda mais a concentração do ácido, o excesso de íons $\mathrm{H}^{+}$solvata a superfície da micela e as forças de repulsão eletrostáticas desestabilizam os agregados, impedindo assim, a separação das fase ${ }^{21}$. Devido à isso, na prática, foi visualizada a turvação da solução.

Este experimento pode ser perfeitamente realizado com o emprego do tensoativo SDS, entretanto, deve ser adicionado $0,1 \% \mathrm{v} / \mathrm{v}$ de Triton X-100 para que a separação das fases ocorra quase que instantaneamente. Desta forma, tanto o SDS quanto o SDSA, promovem a separação das fases. Quando empregado o SDSA, o tempo

Tabela 1. Influência da concentração de ácido sobre a separação por meio do Ponto Nuvem

\begin{tabular}{cc}
\hline $\mathrm{HCl}\left(\mathrm{mol} \mathrm{L}^{-1}\right)$ & $\mathrm{SDSA}(1 \% \mathrm{~m} / \mathrm{v})$ \\
\hline 1 & $\mathrm{~N}$ \\
2 & $\mathrm{~N}$ \\
3 & $\mathrm{~F}$ \\
4 & $\mathrm{~F}$ \\
5 & $\mathrm{~N}^{*}$ \\
\hline
\end{tabular}

$\mathrm{N}$ : não ocorre a separação de fases

$\mathrm{N}^{*}$ : não ocorre a separação de fases ( $c a .4 \mathrm{~h}$ de observação)

F: ocorre a separação de fases para isto era diminuto, dependendo somente da concentração ácida do meio. Já para o SDS, mesmo com o emprego de $\mathrm{HCl} 4 \mathrm{~mol} \mathrm{~L}{ }^{-1}$, era necessário o emprego de banho termostatizado $\left(\mathrm{ca} .50^{\circ} \mathrm{C}\right.$ por $\left.1 \mathrm{~h}\right)$ ou um tempo de repouso de $c a$. 24 h para a completa separação das fases. Como o SDS (aniônico) pode formar uma micela mista com o Triton X-100 (não-iônico), a etapa de aquecimento ou o intervalo de espera podem ser minimizados, visto que foram obtidos tempos semelhantes na separação de fases em comparação ao SDSA.

A micela formada quando empregados SDS e Triton X-100, possui uma concentração maior de SDS do que de Triton X-100. Além disso, nas condições experimentais utilizadas, sem a adição de SDS não ocorria a separação de fases, ao passo que sem a adição de Triton X-100, esta separação era visível, embora em um tempo muito maior. Isto leva a crer que o processo de separação é comandado pelo SDS, embora o Triton X-100 possua um papel importante neste caso, uma vez que induz a um aumento na cinética de separação das fases.

A escolha pelo SDS em detrimento ao SDSA, se deveu ao fato de que, o primeiro, apresenta custo muito menor do que o SDSA, facilitando a sua aquisição e a utilização em aulas práticas. Entretanto, qualquer um dos surfactantes pode ser empregado para esse fim.

Como sugestão de discussão em sala de aula, podem ser enfocados os temas de formação de micelas hidrofílicas/hidrofóbicas, dupla camada elétrica, tratamento de água por floculação, sendo que, inclusive, o docente pode relacionar esses conceitos com alguns produtos de uso diário (ex.: detergentes, maionese, óleos, entre outros) $)^{22-24}$.

\section{Segunda parte: Pré-concentração}

Na segunda parte do primeiro experimento, é enfatizada a pré-concentração de cobalto em função da concentração do tensoativo, bem como a aplicação de algumas características analíticas do método.

O motivo pelo qual se justifica a extração do complexo na fase denominada rica (fase concentrada em tensoativo) se refere a que a relação entre o volume da fase rica e o volume total é minimizada (geralmente entre 1 a 10\% do volume final), propiciando, desta forma, bons fatores de pré-concentração. Os mecanismos envolvidos são semelhantes àqueles empregados na extração líquido-líquido ${ }^{13,19}$. A Figura 1 mostra uma sequiência de fotos obtidas durante a separação de fases usando 4,7 mL de água deionizada, $1 \mathrm{~mL}$ de uma solução padrão de $10 \mathrm{mg} \mathrm{L}^{-1}$ de Co, 0,5 mL de 5-Br-PADAP 0,025\% m/ $\mathrm{v}, 1 \% \mathrm{~m} / \mathrm{v}$ de SDS e 13,3 mL de $\mathrm{HCl} 1: 1 \mathrm{v} / \mathrm{v}$. A fase rica se forma na parte superior do tubo devido a menor densidade do surfactante predominante (SDS - aniônico). Para surfactantes não-iônicos a fase rica normalmente se forma na parte inferior, devido a maior densidade destes surfactantes. Todos estes experimentos foram realizados em duplicata.

Com os resultados obtidos, foram determinados os fatores de pré-concentração para cada situação (Tabela 2). Deve-se ressaltar que o cálculo do fator de pré-concentração teórico é realizado pela simples medida das fases rica e pobre, conforme a equação (1).

$$
F C T=\frac{h_{F P}}{h_{F R}}
$$

Onde: FCT - fator de pré-concentração teórico, $\mathrm{h}_{\mathrm{FP}}$ - altura da fase pobre $(\mathrm{cm})$ $\mathrm{h}_{\mathrm{FR}}$ - altura da fase rica $(\mathrm{cm})$ (2).

Já o fator de pré-concentração real é obtido através da equação

$$
F C R=\frac{[M]_{F R}}{[M]_{\text {In }}}
$$


Tabela 2. Efeito da concentração de SDS na pré-concentração de Co por Ponto Nuvem

\begin{tabular}{|c|c|c|c|c|c|c|c|c|}
\hline [SDS] $(\%)$ & $\begin{array}{c}\text { h-FR }{ }^{\mathrm{a}} \\
(\mathrm{cm})\end{array}$ & $\begin{array}{l}\text { h-FP } \\
(\mathrm{cm})\end{array}$ & $\mathrm{FCT}^{\mathrm{c}}$ & Dil. $^{\mathrm{d}}$ & $\begin{array}{l}{[\mathrm{Co}]_{\mathrm{FR}}^{\mathrm{e}}} \\
\left(\mathrm{mg} \mathrm{L}^{-1}\right)\end{array}$ & $\begin{array}{l}{[\mathrm{Co}]_{\mathrm{FR}}^{\mathrm{f}}} \\
\left(\mathrm{mg} \mathrm{L}^{-1}\right)\end{array}$ & $\begin{array}{l}{[\mathrm{Co}]_{\text {In. }^{\mathrm{g}}}} \\
\left(\mathrm{mg} \mathrm{L}^{-1}\right)\end{array}$ & $\mathrm{FCR}^{\mathrm{h}}$ \\
\hline 0,5 & 0,4 & 13,7 & 34,2 & 3,0 & 2,9 & 8,7 & 0,5 & 17,3 \\
\hline 1,0 & 0,8 & 13,5 & 16,9 & 2,5 & 1,9 & 4,8 & 0,5 & 9,8 \\
\hline 2,0 & 1,6 & 12,7 & 7,9 & 2,3 & 0,8 & 1,8 & 0,5 & 3,7 \\
\hline 3,0 & 2,9 & 11,6 & 4,0 & 2,5 & 0,5 & 1,2 & 0,5 & 2,4 \\
\hline
\end{tabular}

${ }^{\mathrm{a}}$ Altura da fase rica em surfactante; ${ }^{\mathrm{b}}$ Altura da fase aquosa; ${ }^{\mathrm{c}}$ Fator de pré-concentração teórico; ${ }^{\mathrm{d}}$ Fator de diluição da fase rica; ${ }^{\mathrm{e}}$ Concentração de Co na fase rica diluída; ${ }^{\mathrm{f}}$ Concentração de Co na fase rica sem diluição; ${ }^{\mathrm{g}}$ Concentração de Co na solução inicial; ${ }^{\mathrm{h}}$ Fator de pré-concentração real

Onde: FCR - fator de pré-concentração real $[\mathrm{M}]_{\mathrm{FR}}$ - concentração obtida do metal na fase rica $\left(\mathrm{mg} \mathrm{L}^{-1}\right)$ $[\mathrm{M}]_{\mathrm{In}}$ - concentração inicial do metal na solução homogênea $\left(\mathrm{mg} \mathrm{L}^{-1}\right)$

Como pode ser observado na Tabela 2, quanto maior a concentração de SDS, maior o volume da fase rica (altura medida) e menores os fatores de pré-concentração teórico (FCT) e real (FCR). Isto se deve ao fato do metal (neste caso, o cobalto) estar distribuído em um número maior de micelas e, consequentemente, menos concentrado. $\mathrm{O}$ aumento do volume da fase rica ocorre devido ao aumento da concentração do tensoativo, já que a fase rica é majoritariamente formada pelo tensoativo. O FCT é o fator máximo de pré-concentração que se pode obter; por isso, o FCT é maior que o FCR. Apesar de se obter melhores FCT e FCR usando-se $0,5 \%$ de SDS, a quantidade de fase rica formada dificulta a coleta da mesma devido ao pequeno volume obtido. Isso pode ocasionar a mistura das fases durante a coleta, refletindo em perda de precisão. Como a sequiência é proposta para uma aula prática, optou-se em trabalhar com uma concentração de tensoativo maior ( $1 \% \mathrm{~m} / \mathrm{v}$ de SDS), em detrimento do fator de pré-concentração, para se evitar a coleta também da fase pobre.

Deve-se salientar que os dados obtidos com relação ao volume de fase rica, se coadunam àqueles de outros experimentos, nos quais também foi observada uma relação direta e crescente entre o volume de fase rica e a concentração empregada de tensoativo ${ }^{25}$.

Além dos fatores comentados, algumas características analíticas para o método, tais como limites de detecção e de quantificação e a precisão (em nível de repetibilidade) foram avaliados neste experimento, e são mostradas na Tabela 3 . O limite de detecção foi calculado como 3 vezes o desvio padrão de 15 medidas da absorbância do branco, dividido pelo coeficiente angular da curva de calibração. $\mathrm{O}$ limite de quantificação foi calculado como 10 vezes o desvio padrão de 15 medidas da absorbância do branco dividido pelo coeficiente angular da curva de calibração ${ }^{26}$.

Como sugestão nesta parte da prática, o docente poderá discutir, além dos parâmetros aqui calculados, alguns outros, tais como: repetibilidade e reprodutibilidade, comentando sobre os cálculos de rejeição de resultados e os diferentes métodos para tal ${ }^{22,23,27}$.

\section{Experimento 2}

\section{Primeira parte: Formação de complexos e equilíbrio químico}

Nesta primeira parte do EXPERIMENTO 2 o objetivo principal foi o de definir o melhor complexante para a pré-concentração de cobalto. $\mathrm{O}$ experimento também foi realizado sem a adição do complexante, enfatizando assim, a necessidade de seu uso. Assim sendo, os mesmos fatores de pré-concentração calculados na segunda parte do EXPERIMENTO 1, foram avaliados também nesta parte. Os resultados podem ser visualizados na Tabela 4.

Sem o uso de complexantes não ocorre a extração e, conseqüentemente, a pré-concentração do cobalto $(\mathrm{FCR} \approx 1,0)$. O metal não possui a capacidade de se ligar ao surfactante, sendo que a ligação/ interação é feita através do complexante (ligante hidrofóbico). O metal se liga ao complexante e este, por sua vez, interage hidrofóbica e/ou eletrostáticamente ${ }^{3}$ com o surfactante. Deve-se ressaltar que mesmo após a adição do $\mathrm{HCl}$, possivelmente o complexo era mantido, já que a cor do complexo (lilás) podia ser visualizada na fase rica, bem como o cobalto não era detectado por FAAS na fase pobre. Como o complexo interage com a parte hidrofóbica da micela (parte interior da micela), provavelmente o ácido não seja capaz de interagir e

Tabela 3. Características analíticas na determinação de Co por FAAS

\begin{tabular}{lcc}
\hline Curva de Calibração & $\mathrm{A}=0,0025+0,04043 \mathrm{C}\left(\mathrm{mg} \mathrm{L}^{-1}\right)$ & $\mathrm{r}=0,9999$ \\
Limite de Deteção & $66 \mu \mathrm{g} \mathrm{L}^{-1}$ & \\
Limite de Quantificação & $219 \mu \mathrm{g} \mathrm{L}{ }^{-1}$ & $0,4-3,6 \%$ \\
RSD & $0,4 \%$ & \\
\hline
\end{tabular}

Tabela 4. Efeito do complexante na pré-concentração de Co por Ponto Nuvem

\begin{tabular}{|c|c|c|c|c|c|c|c|c|}
\hline Complexante & $\begin{array}{l}\text { h-FR } \\
(\mathrm{cm})\end{array}$ & $\begin{array}{l}\text { h-FP } \\
(\mathrm{cm})\end{array}$ & FCT & Dil. & $\begin{array}{l}{[\mathrm{Co}]_{\mathrm{FRD}}} \\
\left(\mathrm{mg} \mathrm{L}^{-1}\right)\end{array}$ & $\begin{array}{c}{[\mathrm{Co}]_{\mathrm{FR}}} \\
\left(\mathrm{mg} \mathrm{L}^{-1}\right)\end{array}$ & $\begin{array}{l}{[\mathrm{Co}]_{\mathrm{In} .}} \\
\left(\mathrm{mg} \mathrm{L}^{-1}\right)\end{array}$ & FCR \\
\hline S/ compl. & 1,0 & 13,2 & 13,2 & 2,5 & 0,2 & 0,5 & 0,5 & 1,0 \\
\hline 5-Br-PADAP & 0,8 & 13,5 & 16,9 & 2,5 & 1,9 & 4,9 & 0,5 & 9,7 \\
\hline PAN & 0,8 & 13,5 & 16,9 & 2,5 & 1,9 & 4,8 & 0,5 & 9,6 \\
\hline PAR & 0,7 & 13,6 & 19,4 & 2,5 & 0,9 & 2,3 & 0,5 & 4,6 \\
\hline
\end{tabular}

siglas conforme a Tab. 2 
protonar o complexante, o que evitaria que o complexo formado fosse destruído ${ }^{28}$.

O fator de pré-concentração real pode depender de vários fatores, tais como: constante de formação do complexo, da estabilidade do complexo formado, da hidrofobicidade do complexante e afinidade do complexo com o tensoativo. Os FCRs obtidos com o uso do 5-Br-PADAP e do PAN foram muito próximos. Já o FCR obtido com o uso do complexante PAR foi bem menor (ca.50\%). Como são complexantes do mesmo grupo, as constantes de complexação e a estabilidade dos complexos formados são semelhantes ${ }^{29}$, não devendo influir de forma tão acentuada no FCR. Assim sendo, acredita-se que a diferença obtida possa ser devida à menor hidrofobicidade do reagente PAR, já que se trata de um sal monossódico solúvel em água. Pelas estruturas dos três complexantes apresentadas na Figura 2 pode se observar essa diferença.

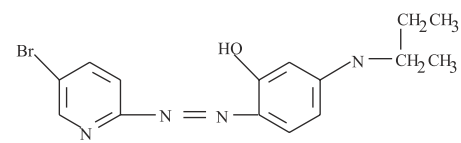

(a)

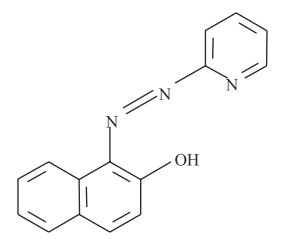

(b)

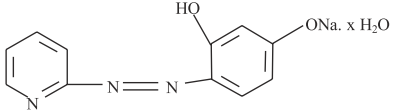

(c)
Figura 2. Estrutura química dos complexantes usados na pré-concentração de Co através do Ponto Nuvem: (a) 5-Br-PADAP; (b) PAN e (c) PAR

Assim sendo, o 5-Br-PADAP foi escolhido para os outros experimentos, sendo que o PAN também poderia ser usado com bons resultados.

Como sugestão para a discussão em classe desta parte da prática, podem ser enfocados os conceitos de complexação, constante de formação, estabilidade de complexos, entre outros ${ }^{22,23,30}$.

\section{Segunda parte: Influência do pH na formação do complexo}

Nessa parte do experimento, procurou-se enfatizar a importância do ajuste do $\mathrm{pH}$ na complexação do cobalto pelo 5-Br-PADAP. Os fatores de pré-concentração foram calculados e são apresentados na Tabela 5.

Embora o FCT seja igual para os dois valores de $\mathrm{pH}$ estudados, verifica-se que o FCR é bem menor (cerca de 1/3) quando não se faz o ajuste do $\mathrm{pH}$. Isto ocorre porque a $\mathrm{pH} 2,5$ a complexação do Co pelo 5-Br-PADAP é menor, e apenas o metal complexado é extraído. Os motivos que levam à isso já foram discutidos anteriormente. Desta forma é de fundamental importância que o pH seja ajustado para a complexação do metal em questão.
A critério do docente, poderá se discutir o efeito do $\mathrm{pH}$ na formação de complexos, protonação e desprotonação de complexantes, cálculo de constantes em função do $\mathrm{pH}$, entre outros $22,23,30$.

\section{Terceira parte: Influência do tempo de complexação}

Nesta última parte, explorou-se o efeito do tempo de complexação (tempo decorrido entre as adições do complexante e a do tensoativo) na eficiência de pré-concentração. Os fatores de pré-concentração foram calculados e os resultados são apresentados na Figura 3. Devese enfatizar que sempre foi empregado excesso na concentração de ligante.

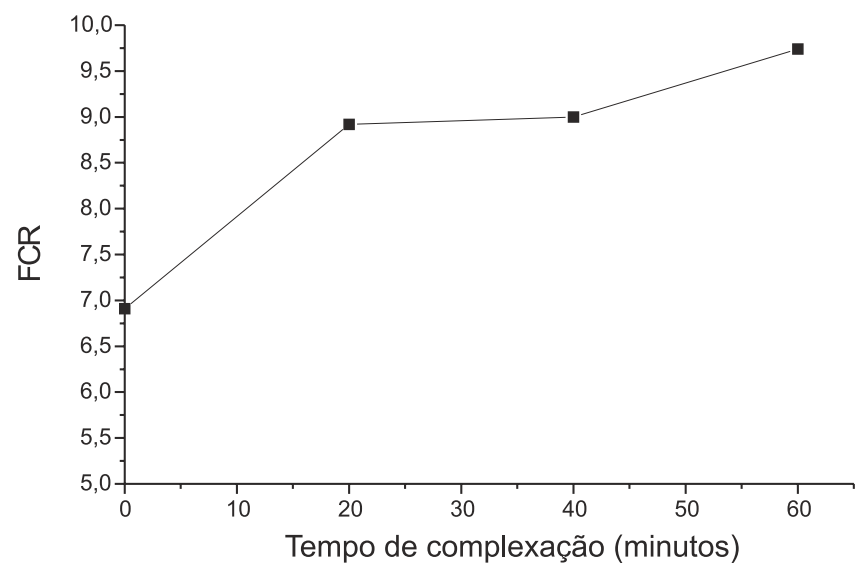

Figura 3. Efeito do tempo de complexação na pré-concentração de Co por Ponto Nuvem. As condições foram aquelas empregadas na Terceira parte do EXPERIMENTO 2

Quando o tempo de complexação é praticamente igual a zero, o FCR é menor $(\approx 7,0)$ indicando que ocorreu a complexação entre o metal e o ligante, entretanto, pode não ter havido tempo suficiente para uma maior formação do complexo Co-5-Br-PADAP. Assim sendo, pode ser que nem todo o cobalto esteja na forma de complexo quando o tensoativo é adicionado. Melhores resultados de FCR são obtidos quando são empregados tempos maiores de complexação. Já com o tempo de $20 \mathrm{~min}$, bons valores de FCR $(\approx 9,0)$ foram encontrados, sendo que este tempo pode ser usado nos outros experimentos. Dependendo da concentração do analito na amostra, tempos menores também poderão ser empregados.

\section{CONCLUSÕES}

Os experimentos propostos neste artigo abordam diferentes assuntos que freqüentemente são discutidos em um curso de química analítica. Da maneira como são apresentados, tentam flexibilizar a aula prática, já que fornecem ao docente, diferentes possibilidades de apresentação da mesma. $\mathrm{O}$ fato dos experimentos serem de fácil visualização, torna a prática ainda muito mais interessante do ponto de vista didático.

Tabela 5. Efeito do pH de complexação na pré-concentração de Co por Ponto Nuvem.

\begin{tabular}{cccccccr}
\hline $\mathrm{pH}$ & $\begin{array}{c}\mathrm{h}-\mathrm{FR} \\
(\mathrm{cm})\end{array}$ & $\begin{array}{c}\mathrm{h}-\mathrm{FP} \\
(\mathrm{cm})\end{array}$ & FCT & Dil. & $\begin{array}{c}{[\mathrm{Co}]_{\mathrm{FRD}}} \\
\left(\mathrm{mg} \mathrm{L}^{-1}\right)\end{array}$ & $\begin{array}{c}{[\mathrm{Co}]_{\mathrm{FR}}} \\
\left(\mathrm{mg} \mathrm{L}^{-1}\right)\end{array}$ & $\begin{array}{c}{[\mathrm{Co}]_{\mathrm{In} .}} \\
\left(\mathrm{mg} \mathrm{L}^{-1}\right)\end{array}$ \\
\hline 2,5 & 0,8 & 13,5 & 16,9 & 2,5 & 0,7 & 1,6 & 0,5 \\
9,0 & 0,8 & 13,5 & 16,9 & 2,5 & 1,9 & 4,9 & 0,5 \\
\hline
\end{tabular}

siglas conforme a Tab. 2 
Se adaptados, os experimentos propostos também poderão ser realizados em disciplina de química geral ou, ainda, na disciplina de química analítica qualitativa, com a vantagem de se poder discutir todos os aspectos já mencionados.

Apesar de ser empregado neste trabalho um espectrômetro de absorção atômica com chama, algumas partes dos experimentos não necessitam de quantificação, e podem perfeitamente ser aplicadas em disciplinas onde não se dispõe deste equipamento.

Outra característica importante deste trabalho, reveste-se no emprego da chamada "química limpa", pois os solventes orgânicos freqüentemente empregados em processos de separação, são substituídos por tensoativos biodegradáveis. Isso vêm de encontro aos objetivos hoje impostos dentro da química, principalmente no que diz respeito à redução da produção de resíduos tóxicos ou de difícil tratamento, dentro de um laboratório de ensino de química.

\section{AGRADECIMENTOS}

Os autores são gratos ao CNPq (Conselho de Desenvolvimento Científico e Tecnológico), pelas bolsas concedidas e à FAPESP (Fundação de Amparo a Pesquisa do Estado de S. Paulo - Projeto n: 99/ 12124-7).

\section{REFERÊNCIAS}

1. Hanson, C.; Recent Advances in Liquid-Liquid Extraction, Ed. Pergamon Press: Oxford, 1975.

2. Sirimanne, S. R.; Barr, J. R.; Patterson Jr, D.G.; Anal. Chem. 1996, 68, 1556.

3. Pelizzetti, E.; Pramauro, E.; Anal. Chim. Acta 1985, 169, 1.

4. Quina, F.H.; Hinze, W.L.; Ind. Eng. Chem. Res. 1999, 38, 4150.

5. Laespada, M.E.F.; Pavón, J.L.P.; Cordero, B.M.; Analyst 1993, 118, 209.

6. Weest, C.C.; Harwell, J.H.; Environ. Sci. Technol. 1992, 26, 2324.

7. Porter, M.R.; Recent Developments in the Analysis of Surfactants. Critical Reports on Applied Chemistry; Ed. Elsevier Science Ltde: New York, 1978, v. 32 .
8. Maniasso, N.; Quim. Nova 2001, 24, 87.

9. Hinze, W.L.; Solution Chemistry of Surfactants, Mittal, K.L., ed., Plenunn Press: New York, 1979.

10. Pinto, C.G.; Pavón, J.L.P.; Cordero, B.M.; Anal. Chem. 1992, 64, 2334.

11. Cordero, B.M.; Pavón, J.L.P.; Pinto, C.G.; Laespada, M.E.F.; Talanta 1993, 40, 1703.

12. Saitoh, T.; Hinze, W.; Anal. Chem. 1991, 63, 2520.

13. Silva, M.A.M.; Frescura, V.L.A.; Aguilera, F.J.N.; Curtius, A.J.; J. Anal. At. Spectrom. 1998, 13, 1369.

14. Silva, M.A.M.; Frescura, V.L.A.; Curtius, A.J.; Spectrochim. Acta, Part B 2000, $55,803$.

15. Casero, I.; Sicilia, D.; Rubio, S.; Perez-Bendito, D.; Anal. Chem. 1999, $71,4519$.

16. Saitoh, T.; Tani, H.; Kamidate, T.; Watanabe, H.; Trends Anal. Chem. 1995, 14, 213.

17. Ferrer, R.; Beltrán, J.L.; Guiteras, J.; Anal. Chim. Acta 1996, 330, 199.

18. Vitouchova, M.; Jancar, L.; Sommer, L.; Fresenius' J. Anal. Chem. 1992, 343, 247.

19. Sicilia, D.; Rubio, S.; Pérez-Bendito, M.D.; Maniasso, N.; Zagatto, E.A.G.; Anal. Chim. Acta 1999, 392, 29.

20. Linfield, W. M. (ed.); Anionic Surfactants, Marcel Dekker, New York, 1976

21. Israelachvili, J.N.; Intermolecular and Surface Forces with Applications to Colloidal and Biological Systems, $3^{\mathrm{a}}$ ed., Academic Press: San Diego, CA, 1989.

22. Baccan, N.; Andrade, J.C.; Godinho, O.E.S.; Barone, J.S.; Química Analítica Quantitativa Elementar, 2a ed., Ed. Edgard Blücher Ltda: São Paulo, 1990.

23. Skoog, D.A.; West, D.M.; Holler, F.J.; Fundamentals of Analytical Chemistry, $6^{\text {a }}$ ed., Sounders College Publishing: EUA, 1992.

24. Atkins, P.W.; Físico-Química, $6^{\mathrm{a}}$ ed., LTC Livros Técnicos Científicos Ed. S/A: Rio de Janeiro, 1997, vol. 2.

25. Maniasso, N.; Tese de Doutorado, Universidade de São Paulo, Brasil, 1999.

26. Analytical Methods Committee; Analyst 1987, 112, 199.

27. Miller, J.C.; Miller, J.N.; Estadística para Química Analítica, 2a ed., Addison-Wesley Iberoamericana: Washington, 1993.

28. Attwood, D.; Florence, A. T.; Surfactants Systems - Their Chemistry, Pharmacy and Biology, $2^{\mathrm{a}}$ ed., Chapman and Hall: New York, 1985.

29. Sandell, E.B.; Onishi, H.; Photometric Determination of Traces of Metals - General Aspects, Ed. John Wiley \& Sons, New York, 1978.

30. Atkins, P.W.; Físico-Química, $6^{\mathrm{a}}$ ed.; LTC Livros Técnicos e Científicos Ed. S/A: Rio de Janeiro, 1997, vol. 1. 